\title{
FIRST-PRINCIPLES STUDY OF SUPERLOW FRICTION BETWEEN HYDROGENATED DIAMOND SURFACES
}

\author{
S. Ciraci ${ }^{1, *}$ and S. Dag ${ }^{1}$ \\ ${ }^{1}$ Department of Physics, Bilkent University, Ankara 06800, Turkey
}

(Dated: June 14, 2005)

\begin{abstract}
Attractive interaction between two clean diamond(001) slabs turns repulsive upon the hydrogenation of surfaces. Even under high loading forces, this repulsive interaction prevents the sliding surfaces from being closer to each other. As a result, calculated lateral force variation generated during sliding has small magnitude under high constant loading forces. Superlow friction observed earlier between diamond like carbon coated surfaces can be understood by the steady repulsive interaction between sliding surfaces, as well as strong and stiff carbon-carbon and carbon-hydrogen bonds which do not favor energy dissipation. In ambient conditions, the steady repulsive interaction is, however, destroyed by oxygenation of hydrogenated surface.
\end{abstract}

\section{INTRODUCTION}

Friction between two surfaces in relative motion is induced by short- and long-ranged interaction between surfaces and may involve phononic, electronic even photonic energy dissipation, quantum energy transport, structural phase transitions and various chemical processes. ${ }^{1}$ In boundary lubrication specific foreign atoms or molecules are placed between sliding surfaces to reduce the friction coefficient by cutting down the strong interaction between surfaces. Recently, Erdemir et al. ${ }^{2}$ reported superlow friction and wear between diamondlike carbon (DLC) coated surfaces produced using a hydrogen-rich plasma. They achieved kinetic friction coefficients $\mu_{k}$, as low as 0.001 and wear rates of $10^{-9}-10^{-10} \mathrm{~mm}^{3} / \mathrm{Nm}$ in inert-gas environments under $10 \mathrm{~N}$ load at $0.2-0.5 \mathrm{~m} / \mathrm{s}$ sliding velocities.

In this paper we report our study of the atomic scale friction between two hydrogenated diamond(001)- $(2 \times 1)$ surface in relative motion by using first-principles pseudopotential plane wave method within the density functional theory ${ }^{3}$.

\section{MODEL AND RESULTS}

Hydrogenated DLC (H:DLC) coating is a complex, amorphous structure showing irregularities; their sliding surfaces cannot be commensurate and contain irregularly distributed asperities. Even if several processes in the sliding friction have stochastic nature, local bond order and C-H bond topology are similar to various hydrogenated diamond surfaces. Therefore, the interaction between H:DLC surfaces and the resulting friction can be understood by the present model. Here two features, namely full relaxation of atoms in the surfaces and accurate calculations of lateral force components under constant loading force $F_{N}$, are of particular importance. Diamond $(001)-(2 \times 1)$ surfaces are represented by two slabs facing each other at a distance, where carbon atoms in first five surface atomic planes are fully relaxed.

As for the interaction ${ }^{4}$ between two diamond(001)$(2 \times 1)$ slabs, strong bonds form between sliding surfaces, when two commensurate slab surfaces are placed at equilibrium separation so that $F_{z} \cong 0$. Once a normal force is applied, $F_{z}$ becomes repulsive since atoms of different surfaces come close to each other at $d<1.5 \AA$. Under these circumstances, $\mu_{k}$, as well as wear rate are expected to be high in the sliding motion, where sequential bond breaking and rebounding take place. Strong interaction between bare diamond $(001)-(2 \times 1)$ surface is shown in Fig. 1(c).

The above situation is, however dramatically different when all C-dangling bonds on two slab surfaces facing each other are saturated by $\mathrm{H}$ atoms to form a monohydride phase i.e. H: diamond $(001)-(2 \times 1)$. A repulsive short-range force $F_{z}$ is induced between hydrogenated surfaces for $d<2.5 \AA$; the variation of it with the separation $d$ is shown in Fig. 1(d). Although the repulsive force is reduced by the Van der Waals attraction between slabs, it still keeps the sliding surfaces wide apart at a distance $d$ to balance the loading force $F_{N}$. As a result sliding surfaces are prevented from being too close to dissipate energy by deforming $\mathrm{C}-\mathrm{H}$ bonds or by bond breakings.

It is important to know whether the repulsive interaction continues to keep surfaces wide apart, if one of the diamond slabs are laterally displaced under different loading forces, $F_{N}$. To this end, we carried out series of ab-initio total energy $E_{T}$, normal force $F_{z}$ and lateral force $F_{L}$ calculations corresponding to different lateral displacements, $\Delta x$ and $\Delta y$. Note that keeping two backends of slabs at a fixed distance $D$ is equivalent to inducing a loading force, $F_{N}(D)$, which, in turn, is balanced 

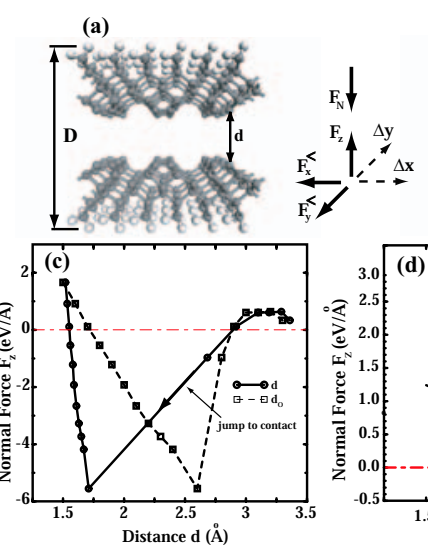

(b) $\quad \mathrm{F}_{\mathrm{N} \downarrow}$

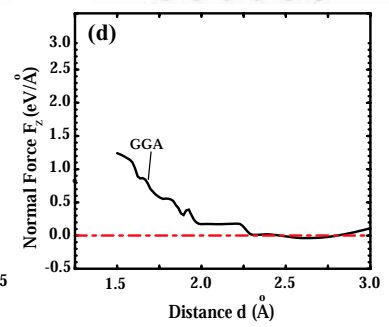

FIG. 1: (a) Two clean diamond(001)- $(2 \times 1)$ surface at a separation $d$. $\mathrm{D}$ is the distance between the back ends of two slabs; $d_{0}$ is the initial separation before the relaxation of slabs; $d$ is the actual separation of the surfaces after relaxation. (b) Two hydrogenated diamond(001)-(2×1) surface at the actual separation $d$. Lateral displacements $(\Delta x, \Delta y)$, lateral force components $\left(F_{x}, F_{y}\right)$, perpendicular force $F_{z}$ and leading force $F_{N}$ are schematically described. Light and dark balls indicate $\mathrm{H}$ and $\mathrm{C}$ atoms, respectively. (c) and (d) Variation of the normal force $F_{z}[\mathrm{eV} / \AA$ per $(2 \times 1)$ cell $]$ as a function of separation $d$ between clean and hydrogenated diamond(001)- $(2 \times 1)$ surfaces, respectively.
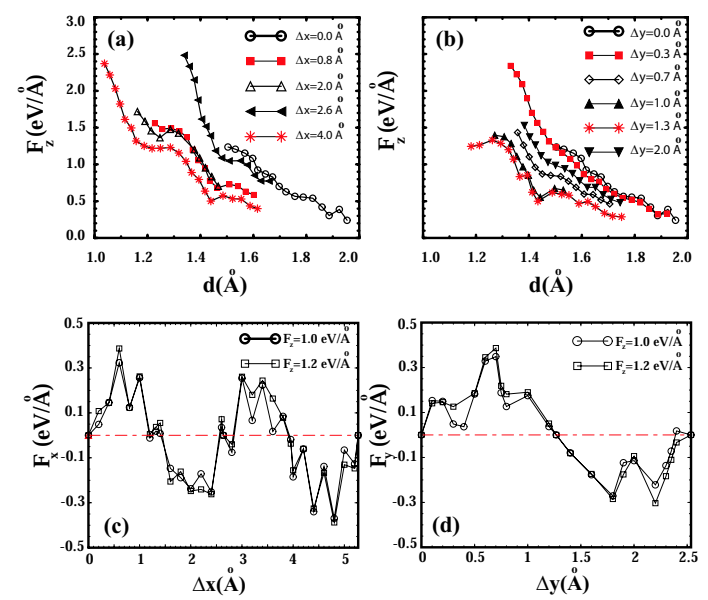

FIG. 2: (a) and (b) Variation of $F_{z}$ as a function of $d$ calculated for different lateral displacements $\Delta x$ and $\Delta y$. (c) and (d) Variation of lateral force $F_{x}$ and $F_{y}$ (in eV/A per $(2 \times 1)$ cell) under constant $F_{N}$ as a function of displacement of the top surface $\Delta x$ and $\Delta y$, respectively. by $F_{z}$. This way, a data base of $E_{T}, F_{z}, F_{L}\left(F_{x}, F_{y}\right)$, and $d$ corresponding to various values of $\Delta x, \Delta y$ and $D$ have been created. The generation of strong repulsive force is the most essential aspect leading to superlow friction and is reminiscent of a boundary lubrication.

In Fig. 2(a) and 2(b) we show the calculated variation of the normal force as a function of $d$ for different lateral displacement of $\Delta x$ and $\Delta y$. Using these curves and related data, we obtain $F_{x}(\Delta x)$ and $F_{y}(\Delta y)$ under a constant $F_{N}$. These variations lead to the well-known stickslip curves in an adiabatic process ${ }^{5}$. Now as an adhoc approach to estimate $\mu_{k}$ in energy dissipating medium we assume that the work done by the lateral force $F_{L}$ (i.e. when it is parallel to the direction of motion, $F_{L}^{>}$) is totally $\operatorname{lost}^{6}$ and calculate average friction force $\overline{F_{f}}$. Using Fig. 2(a) we found $\overline{F_{f}} \sim 0.05 \mathrm{eV} / \AA$ for $F_{N}=1 \mathrm{eV} / \AA$, and $\overline{F_{f}} \sim 0.07 \mathrm{eV} / \AA$ for $F_{N}=1.2 \mathrm{eV} / \AA$. The corresponding kinetic friction constant is $\mu_{k} \sim 0.05$ for both cases. For the reasons discussed above, more realistic estimation could be obtained from $\overline{F_{f}}=\int\left(F_{x}^{<}-F_{x}^{>}\right) d x / R$, if lateral force variation were calculated precisely. Our crude force variation in Fig. 2 (a) yields $\mu_{k} \sim 0.01$.

Finally, we address to the issue why hydrogenated DLC films can be unstable in atmospheric conditions. In order to test the stability of the hydrogenated surface under ambient condition, we placed $\mathrm{O}$ atom at different sites of the H:diamond(001)- $(2 \times 1)$. Upon relaxation $\mathrm{O}$ has been adsorbed. Favorably, it attacked the $\mathrm{C}-\mathrm{H}$ bonds by entering between $\mathrm{C}$ and $\mathrm{H}$ atoms to form $\mathrm{C}-\mathrm{O}-\mathrm{H}$ radicals. This way, the steady repulsive interaction between surfaces prior to the oxygenation has ceased to destroy superlow friction.

In conclusion, we modelled the sliding friction between hydrogenated diamond(001)- $(2 \times 1)$ surfaces, and revealed important ingredients leading to superlow friction: These are (i) repulsive interaction between sliding surfaces generated by hydrogenation which persists at any relative position of these surfaces and is strong even at large distance to prevent $\mathrm{C}-\mathrm{H}$ bonds of disordered surfaces from merging; (ii) strong and stiff $\mathrm{C}-\mathrm{H}$ bonds and stiff diamond crystal itself preventing excessive energy from dissipation. It is found that oxygenation of surfaces in the atmospheric conditions destroys the steady repulsive interaction.
* Electronic address: ciraci@fen.bilkent.edu.tr

1 B. N. J. Persson, Sliding Friction: Physical principles and Applications (Springer-Verlag, Berlin 2000).

2 A. Erdemir, O.L. Eryilmaz and G. Fenske, J. Vac. Sci. Technol. A18(4), 1987 (2000).

3 S. Dag and S. Ciraci, Phys. Rev. B 70, 241401(R) (2004).

${ }^{4}$ S. Ciraci et al., Phys. Rev. B 42, 7618 (1990); ibid46, 10411
(1992).

5 G.A. Tomlinson, Philos Mag. 7, 905 (1929).

${ }^{6}$ W. Zhong and D. Tomanek, Phys. Rev. Lett. 64, 3054 (1990); D. Tomanek, W. Zhong, and H. Thomas, Europhys. Lett. 15, 887 (1991). 